\title{
Using Green's functions and split Gaussian integration for two-point boundary value problems on the ray
}

\author{
$\begin{array}{ll}\text { G. D. McBain } & \text { S. W. Armfield } \\ & \end{array}$
}

(Received 31 August 2006; revised 10 April 2007)

\begin{abstract}
We continue our study of the construction of numerical methods for solving two-point boundary value problems using Green's functions, building on the successful use of product integration to achieve the convergence expected of Gauss-type quadrature schemes. We introduce refinements such as the use of cardinal basis functions to eliminate the need for a transformation from the ordinates to the expansion coefficients. For problems on the ray, we algebraically map the ray to a segment, and there use (cardinal) Legendre polynomials for interpolation and Gauss's rule for quadrature. Numerical examples (the heat and Burgers equations) demonstrate the applicability of the method to problems on the ray, particularly for the sequence of twopoint boundary value problems arising from constant time-stepping for parabolic evolution problems; nonlinear terms, as in the Burgers equation, present no special difficulty.
\end{abstract}

See http://anziamj.austms.org.au/ojs/index.php/ANZIAMJ/article/view/126 for this article, (c) Austral. Mathematical Soc. 2007. Published April 27, 2007. ISSN $1446-8735$ 


\section{Contents}

1 Introduction

C35

1.1 Constant time-stepping for parabolic evolution . . . . . . C36

1.1.1 Explicit time-stepping for nonlinear terms . . . . C C

2 The method

C37

2.1 Primary abscissae . . . . . . . . . . . . . . . . C C39

2.2 Quadrature rules .................... . . . C39

2.3 Evaluating the sampling functions . . . . . . . . . C40

3 Examples

$\mathrm{C} 40$

3.1 A simple example . . . . . . . . . . . . . . . . C C41

3.2 Development of anabatic wind . . . . . . . . . . . C C42

3.3 Vertical moisture infiltration through soil . . . . . . . . C44

4 Conclusion

C46

References

C46

\section{Introduction}

The solution of a linear two-point boundary value problem generally has a Green's integral representation [7, pp. 254-257; e.g.]; for example,

$$
u(x)-\lambda^{-2} u_{x x}(x)=f(x),
$$

for $u \sim 0$ as $x \rightarrow \infty$ and $u(0)$ or $u_{x}(0)$ specified, respectively, has solutions

$$
\begin{aligned}
& u(x)=u(0) \mathrm{e}^{-\lambda x}+\int_{0}^{\infty} \frac{\lambda}{2}\left\{\mathrm{e}^{-\lambda\left|x-x^{\prime}\right|}-\mathrm{e}^{-\lambda\left(x+x^{\prime}\right)}\right\} f\left(x^{\prime}\right) \mathrm{d} x^{\prime}, \\
& u(x)=-\frac{u_{x}(0)}{\lambda} \mathrm{e}^{-\lambda x}+\int_{0}^{\infty} \frac{\lambda}{2}\left\{\mathrm{e}^{-\lambda\left|x-x^{\prime}\right|}+\mathrm{e}^{-\lambda\left(x+x^{\prime}\right)}\right\} f\left(x^{\prime}\right) \mathrm{d} x^{\prime},
\end{aligned}
$$


provided the forcing function $f$ is such that the integrals converge.

Here we continue our development [12] of numerical methods for two-point boundary value problems based on this representation of the solution. Although the basic approach is in principal applicable to unbounded intervals, all the examples in the earlier work dealt with finite intervals, and unboundedness does pose some technical difficulties; for example, the integral of the Green's function $G$ with the force distribution $f$,

$$
\int_{a}^{b} G\left(x, x^{\prime}\right) f\left(x^{\prime}\right) \mathrm{d} x^{\prime}
$$

immediately becomes improper. Here we focus on the ray $0<x<\infty$, as it occurs in a number of models arising in hydrodynamics - buoyancydriven [11] and aerodynamic boundary layers, and vertical infiltration of moisture in soil [3]. We also describe recent refinements to the method.

\subsection{Constant time-stepping for parabolic evolution}

The trapezoidal rule $[9$, pp. 15,250$]$ reduces the parabolic problem

$$
u_{t}=\mathcal{L} u,
$$

to the sequence of two-point boundary value problems

$$
\mathcal{A} u^{k}=f^{k},
$$

where

$$
\begin{aligned}
\mathcal{A} & \equiv 1-\frac{\Delta t}{2} \mathcal{L}, \\
f^{k} & \equiv\left(1+\frac{\Delta t}{2} \mathcal{L}\right) u^{k-1},
\end{aligned}
$$

and the $k$ superscript means at time $t=k \Delta t$. For the heat equation with constant thermal diffusivity $\nu, \mathcal{L} u=\nu u_{x x}$ and each time-step is (1) with $\lambda^{2}=2 / \nu \Delta t$. 


\subsubsection{Explicit time-stepping for nonlinear terms}

Nonlinear terms on the right-hand side of (4) can be integrated explicitly; for example, the second-order Adams-Bashforth scheme [9, p. 41] converts $u_{t}=$ $\mathcal{L} u+\mathcal{N} u$ again to $\mathcal{A} u^{k}=f^{k}$ but with $f^{k}$ in (7) augmented by $\frac{\Delta t}{2}\left(3 \mathcal{N} u^{k-1}-\right.$ $\mathcal{N} u^{k-2}$ ); that is,

$$
\left(1-\frac{\Delta t}{2} \mathcal{L}\right) u^{k}=\left(1+\frac{\Delta t}{2} \mathcal{L}\right) u^{k-1}+\frac{\Delta t}{2}\left(3 \mathcal{N} u^{k-1}-\mathcal{N} u^{k-2}\right) .
$$

\section{The method}

As pointed out earlier [12], the primary difficulty in using (3) for numerical evaluation of the solution is that the Green's function is not smooth over the ridge $x^{\prime}=x$; if the coefficients are smooth and the equation is of order $k$, the Green's function only has $k-2$ continuous partial derivatives with respect to $x$ [7, p. 255; e.g.]. This lack of smoothness carries over to the integrand of (3) and prevents, for example, Gaussian integration from achieving its usual rapid convergence. The basic remedy is to split the integral (3) at $x^{\prime}=x$ :

$$
\int_{a}^{b} G\left(x, x^{\prime}\right) f\left(x^{\prime}\right) \mathrm{d} x^{\prime}=\int_{a}^{x} G\left(x, x^{\prime}\right) f\left(x^{\prime}\right) \mathrm{d} x^{\prime}+\int_{x}^{b} G\left(x, x^{\prime}\right) f\left(x^{\prime}\right) \mathrm{d} x^{\prime} .
$$

We assume that $G$ is known and can be evaluated anywhere, but that in general we will only have incomplete knowledge about $f$, say because, as in (7), it is only computed from approximate solutions at previous time steps.

Say we want to approximate (9) at a set of primary abscissae,

$$
\left\{x_{i}: a \leq x_{1}<x_{2}<\cdots<x_{n} \leq b\right\},
$$


using quadrature rules. Note that each of these $2 n$ integrals has a different domain, so that the quadrature weights and abscissae will depend on the subscript $i$; that is, on the point at which Green's integral is being evaluated. If $f$ is only known at the primary abscissae (10), evaluating the quadrature rules will require interpolation onto certain secondary abscissae.

Say, for the set of primary abscissae (10), we have the sampling functions $\left\{\phi_{i}\right\}_{i=1}^{n}$ that can be evaluated anywhere with the property that $\phi_{j}\left(x_{i}\right)=\delta_{i j}$ that is, $\phi_{j}\left(x_{i}\right)=1$ if $j=i$ and zero otherwise - then the function $f$ can be interpolated given its ordinates with

$$
\hat{f}(x) \equiv \sum_{j} \phi_{j}(x) f\left(x_{j}\right)
$$

and (9) can be approximated by the product integration formula [12]

$$
\int_{a}^{b} G\left(x_{i}, x^{\prime}\right) f\left(x^{\prime}\right) \mathrm{d} x^{\prime} \doteq \sum_{j} g_{i j} f\left(x_{j}\right),
$$

where

$$
g_{i j} \equiv \sum_{k}\left[w_{i k}^{L} G\left(x_{i}, x_{i k}^{L}\right) \phi_{j}\left(x_{i k}^{L}\right)+w_{i k}^{R} G\left(x_{i}, x_{i k}^{R}\right) \phi_{j}\left(x_{i k}^{R}\right)\right]
$$

in which the $w_{i k}^{L}$ and $x_{i k}^{L}$ are the $k$ th weight and abscissa for the quadrature rule on the left subinterval from $a$ to $x_{i}$, and similarly for $w_{i k}^{L}$ and $x_{i k}^{R}$ for the right subinterval from $x_{i}$ to $b$.

In summary, the method is: for a given interval, choose the primary abscissae in (10), the left and right quadrature rules in (13), and the sampling functions in (11), and evaluate the latter at the secondary abscissae. For a given differential operator, find the Green's function and evaluate it for $x$ at the primary abscissae and $x^{\prime}$ at the secondary abscissae to form the matrix of coefficients in (13). For a given forcing function, multiply its column of ordinates by the matrix of coefficients, as in (12). 
The remainder of this section covers good choices for the primary abscissae $(\S 2.1)$ and quadrature rules ( $(2.2)$, and reliable routines for interpolating the sampling functions at the secondary abscissae $(\S 2.3)$.

\subsection{Primary abscissae}

The primary abscissae should be chosen so that the ordinates there represent the function well; for example, for interpolation. For finite intervals, it is well known that equally spaced points lead to poor interpolants; Chebyshev and Gauss points work much better [1]. However, for the ray, polynomial interpolation is inappropriate if the function is supposed to decay (to zero or a constant) far from the origin, since no polynomials beyond the first degree do this.

An alternative is to map the ray to a segment and use polynomial interpolation there; for example, to $-1<t<1$ using (for some $L>0 ; L=1$ hereafter) [6]

$$
t(x)=\frac{x-L}{x+L}, \quad x(t)=L \frac{1+t}{1-t} .
$$

We take the primary abscissae as the Gauss points in $-1<t<1$.

\subsection{Quadrature rules}

Another difference in problems on the ray from those on finite segments is that the split Green's integral (9) requires quadrature rules for both finite segments $\left(a<x^{\prime}<x_{i}\right)$ and right-unbounded segments $\left(x_{i}<x^{\prime}<\infty\right)$.

Gauss's rule, $\int_{-1}^{1} f(t) \mathrm{d} t \doteq \sum_{k} q_{k} f\left(t_{k}\right)$, can be used for both finite and right-infinite intervals using affine and algebraic plus affine mappings;

$$
\int_{0}^{x_{i}} f\left(x^{\prime}\right) \mathrm{d} x^{\prime} \doteq \sum_{k} w_{i k}^{L} f\left(x_{i k}^{L}\right), \quad \int_{x_{i}}^{\infty} f\left(x^{\prime}\right) \mathrm{d} x^{\prime} \doteq \sum_{k} w_{i k}^{R} f\left(x_{i k}^{R}\right),
$$


where

$$
\begin{aligned}
w_{i k}^{L} & =\frac{x_{i} q_{k}}{2}, & w_{i k}^{R} & =\frac{2 L q_{k}}{\left(1-t_{k}\right)^{2}}, \\
x_{i k}^{L} & =\frac{x_{i}\left(t_{k}+1\right)}{2}, & x_{i k}^{R} & =x_{i}+L \frac{1+t_{k}}{1-t_{k}} .
\end{aligned}
$$

Although they are tabulated, the Gauss weights and abscissae were here conveniently computed from the Jacobi matrix [10].

\subsection{Evaluating the sampling functions}

If the primary abscissae (10) go under the mapping (14) to $t_{i}=t\left(x_{i}\right)$, and the $\phi_{i}(t)$ are their sampling polynomials, then a function $f(x)$ on $0<x<\infty$ can be stably interpolated on $-1<t<1$ using the barycentric formula [1]

$$
\begin{aligned}
& \hat{f}(t)=\left[\sum_{m=1}^{n} \frac{w_{m}}{t-t_{m}}\right]^{-1}\left[\sum_{m=1}^{n} \frac{w_{m} f\left(t_{m}\right)}{t-t_{m}}\right], \\
& \text { where } \quad w_{i} \equiv\left[\prod_{j=1, j \neq k}^{n}\left(t_{k}-t_{j}\right)\right]^{-1} .
\end{aligned}
$$

The sampling polynomials, having the property $\phi_{j}\left(t_{i}\right)=\delta_{i j}$, and being identical to their interpolants, can be evaluated at an arbitrary $t$ as

$$
\phi_{j}(t)=\frac{\omega_{j}(t)}{\sum_{m=1}^{n} \omega_{m}(t)}, \quad \text { where } \quad \omega_{j}(t) \equiv \frac{w_{j}}{t-t_{j}} .
$$

\section{$3 \quad$ Examples}

The method developed in $\S 2$ has been implemented in GNU Octave [5]. 


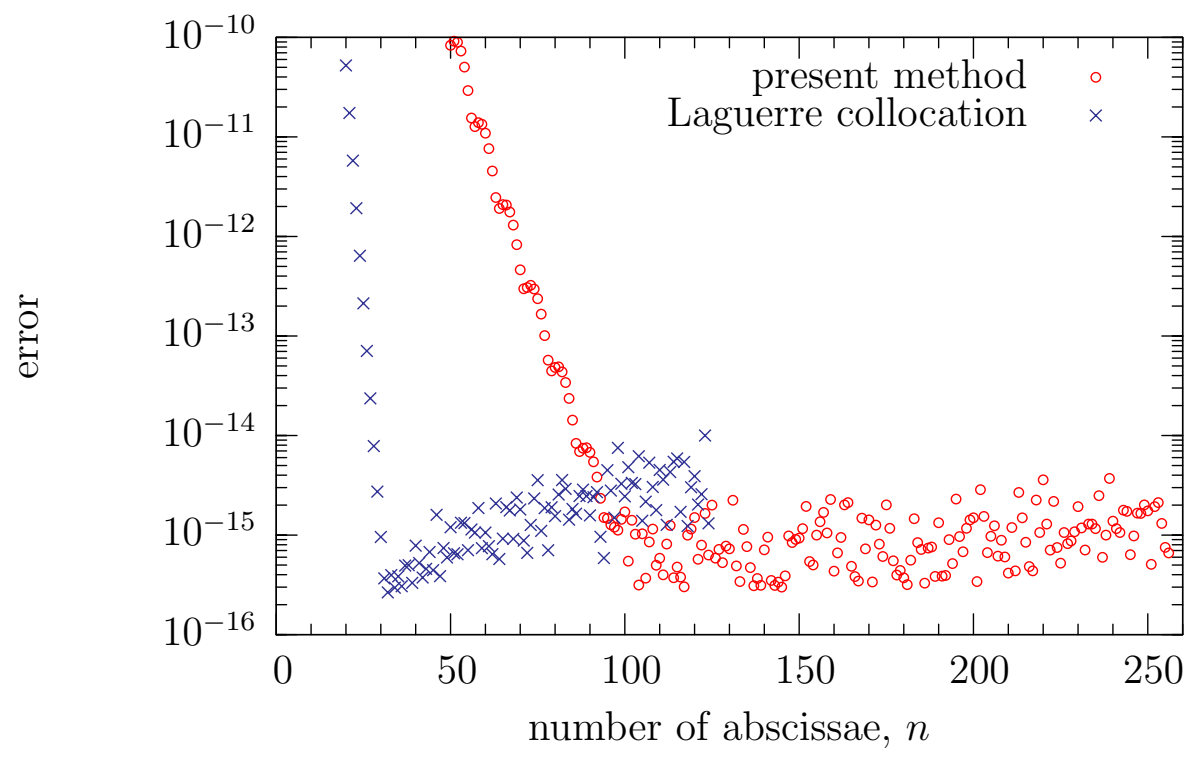

Figure 1: Convergence of the present method and Laguerre collocation for the two-point boundary value problem $u_{x x}-u=-2 \mathrm{e}^{-x}$ with boundary conditions $u(0)=u(\infty)=0$.

\subsection{A simple example}

For $\lambda=1$ and $f(x)=2 \mathrm{e}^{-x}$, the solution of (1) satisfying $u(0)=0$ is $u(x)=x \mathrm{e}^{-x}$. The convergence of the method is excellent: the logarithm of the error (assessed as the maximum absolute error at the abscissae in this example) decreases almost linearly with $n$, as shown in Figure 1.

Moreover, the method seems to remain stable as $n$ increases; something which cannot be said of another spectral-type approach: ordinate-based pseudospectral differentiation matrices [14] based on generalized Laguerre polynomials [11]. As also shown in Figure 1, the collocation method converges faster for $n<30$ but is not stable; the error increases up to $n=124$, with the errors exceeding those for the present method for $n>100$. The collo- 
cation method fails for $n \geq 125$ as the differentiation matrices become so ill-conditioned as to be numerically singular. This drawback of Laguerre collocation is well known: indeed, Iranzo \& Falqués [8] reported that it was only 'possible to apply it reliably up to $N=19$ '.

\subsection{Development of anabatic wind}

The development of the buoyancy layer on an evenly heated vertical wall in a stratified fluid of unit Prandtl number is governed by [13]

$$
\begin{aligned}
& 2 v_{t}=v_{x x}+2 T, \\
& 2 T_{t}=T_{x x}-2 v,
\end{aligned}
$$

subject to $v(0, t)=v(\infty, t)=T(\infty, t)=v(x, 0)=T(x, 0)=0$ and $T_{x}(0, t)=$ -1 . The wall temperature evolves as

$$
T(0, t)=2 C(\sqrt{2 t / \pi})-\sin t / \sqrt{\pi t / 2},
$$

where $C(z)$ is the Fresnel cosine integral [13]. We solved this using the explicit scheme of $\S 1.1 .1$ to uncouple $v$ and $T$ in (21).

The derivatives on the right-hand sides were computed using pseudospectral differentiation matrices. The $k$ th derivative of the interpolant (11) at $x=x_{i}$ is

$$
\hat{f}^{(k)}\left(x_{i}\right)=\sum_{j} \phi_{j}^{(k)}\left(x_{i}\right) f\left(x_{j}\right)
$$

that is, the product of the $k$ th pseudospectral differentiation matrix with coefficients $\phi_{j}^{(k)}\left(x_{i}\right)$ and the column of ordinates $f\left(x_{j}\right)$. Algorithms for constructing differentiation matrices are described by Weideman \& Reddy [14].

The error in $T(0,1)$ (Figure 2) shows rapid spatial convergence, with the error dominated for sufficiently large $n$ by the second-order time-stepping (with the $n$ required increasing with decreasing $\Delta t$ ). 


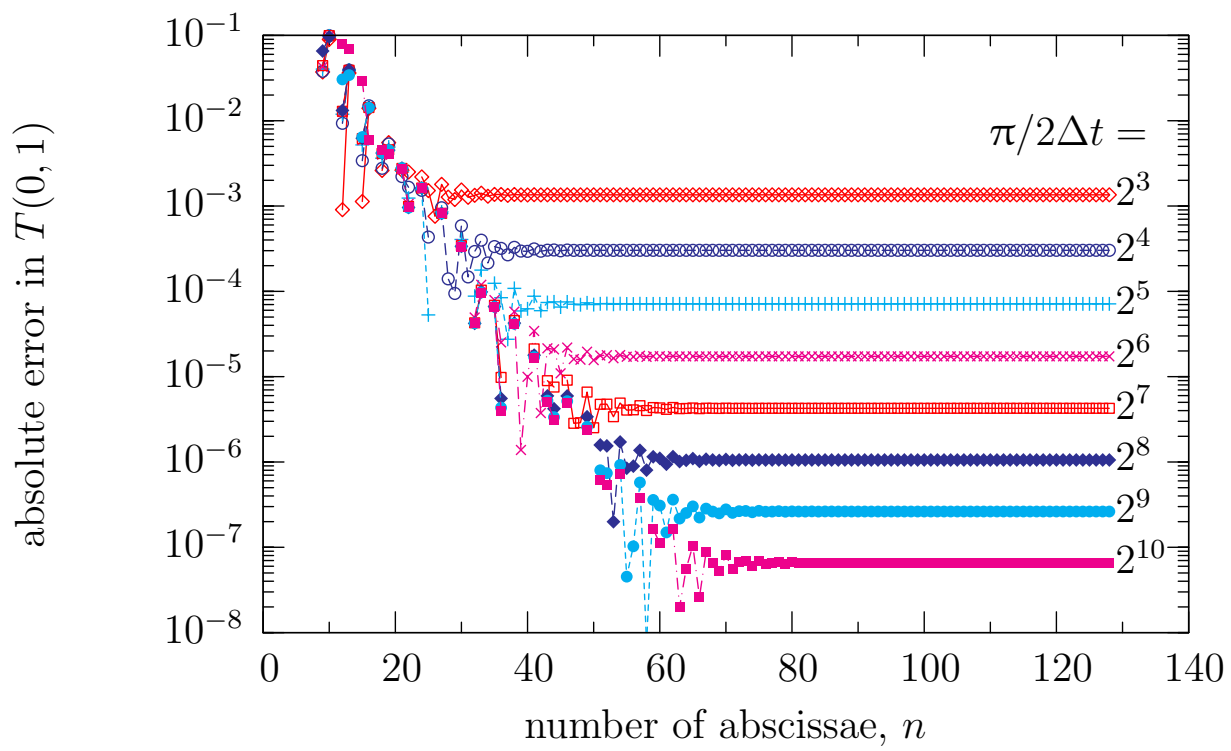

Figure 2: Convergence in $n$ and $\Delta t$ of the method for the example of $\S 3.2$. 


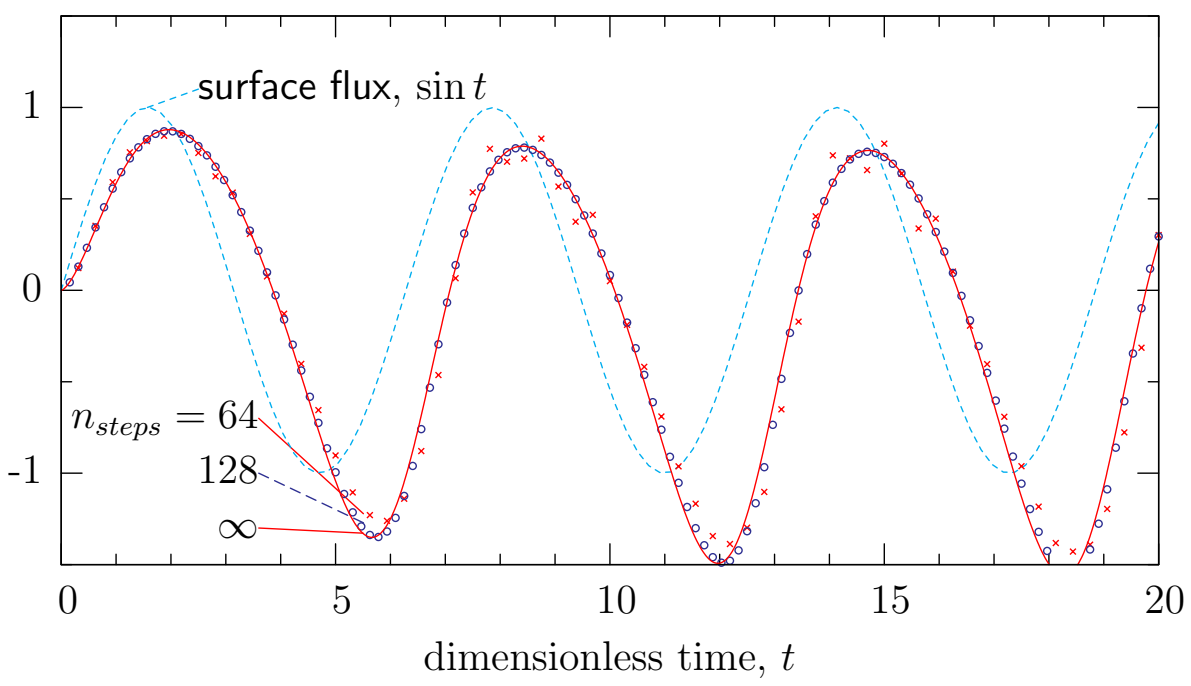

Figure 3: Surface value following surface flux in the Burgers problem (§ 3.3).

\subsection{Vertical moisture infiltration through soil}

The explicit time-stepping of $\S 1.1 .1$ also extends the method to quasilinear problems, such as the Burgers equation with surface flux

$$
\begin{aligned}
u_{t} & =\nu u_{x x}-2 u u_{x}, & & (0<x) \\
u_{x}-u^{2} & =-g(t), & & (x=0)
\end{aligned}
$$

which models vertical infiltration of moisture through soil [3].

The time-discretized version (8) of (23a) is

$$
u^{k}-\frac{\nu \Delta t}{2} u_{x x}^{k}=u^{k-1}+\frac{\nu \Delta t}{2} u_{x x}^{k-1}-\Delta t\left(3 u^{k-1} u_{x}^{k-1}-u^{k-2} u_{x}^{k-2}\right) \text {. }
$$

The nonlinear term in (23b) is extrapolated from the last two values, to retain second-order accuracy in $\Delta t$, so the discrete surface condition for (2b) 


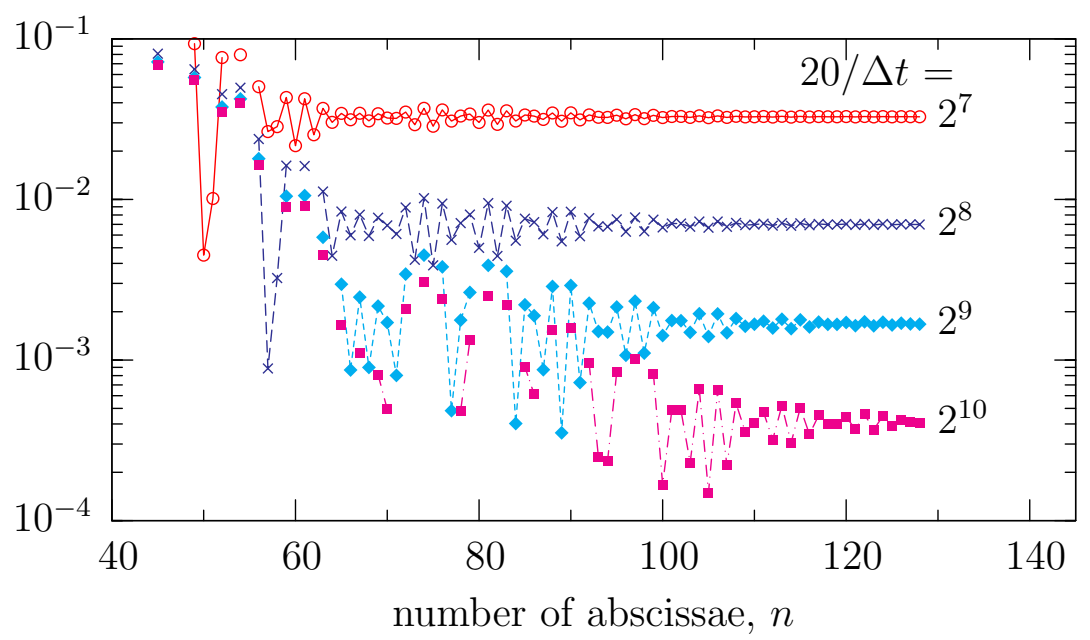

FiguRE 4: Error in the surface value at $t=20$ for the Burgers problem. 
is

$$
u_{x}^{k}(0)=\left\{2 u^{k-1}(0)-u^{k-2}(0)\right\}^{2}-g(k \Delta t) .
$$

Here we solve for $g(t)=\sin t$, as in a published finite difference study [2]. Figures 3-4 compare the value at $x=0$ with the exact solution $[2,4]$

$$
u(0, t)=\frac{\sqrt{2 t / \pi}}{c(t)} \int_{0}^{1} \frac{g(t s) c(t s) \mathrm{d} s}{\sqrt{1-s}} \text { where } c(t) \equiv \exp \left\{\int_{0}^{t} g(s) \mathrm{d} s\right\},
$$

which is accurately evaluated using a Gauss-Jacobi rule [10]. Again, rapid spatial and second-order temporal convergence is attained (Figure 4).

\section{Conclusion}

Green's functions can be used to solve two-point boundary value problems on the ray by splitting the integral along the ridge of the Green's function and using product integration with, for example, cardinal Legendre polynomials algebraically mapped to the ray. The method is effective when many righthand sides are to be solved for the same ordinary differential operator; for example, as in constant time-stepping. The method converges rapidly and appears to be more stable than Laguerre collocation.

\section{References}

[1] J.-P. Berrut and L. N. Trefethen. Barycentric Lagrange interpolation. SIAM Rev., 46:501-517, 2004. MathReview:2115059 C39, C40

[2] G. Biondini and S. de Lillo. Semiline solutions of the Burgers equation with time dependent flux at the origin. Phys. Lett. A, 220:201-204, 1996. MathReview:1406959 C46 
[3] B. E. Clothier, J. H. Knight, and I. White. Burgers' equation: Application to field constant-flux infiltration. Soil Sci., 132(4):99-261, 1981. C36, C44

[4] J. Davoudi and S. Rouhani. PDFs of the Burgers equation on the semiline with fluctuating flux at the origin. Phys. Lett. A, 257:158-164, 1999. doi:10.1016/S0375-9601(99)00285-6 C46

[5] http://www.octave.org C40

[6] C. E. Grosch and S. A. Orszag. Numerical solution of problems in unbounded regions: Coordinate transforms. J. Comput. Phys., 25:273-296, 1977. MathReview:0488870 C39

[7] E. L. Ince. Ordinary Differential Equations. Dover, New York, 1956. MathReview:0010757 C35, C37

[8] V. Iranzo and A. Falqués. Some spectral approximations for differential equations in unbounded domains. Comput. Methods Appl. Mech. Engrg, 98(1):105-126, 1992. MathReview:1172676 C42

[9] J. D. Lambert. Computational Methods in Ordinary Differential Equations. Wiley, London, 1973. MathReview:0423815 C36, C37

[10] D. P. Laurie. Computation of Gauss-type quadrature formulas. J. Comput. Appl. Math., 127:201-217, 2001. MathReview:1808574 C40, C46

[11] G. D. McBain and S. W. Armfield. Linear stability of natural convection on an evenly heated vertical wall. In M. Behnia, W. Lin, and G. D. McBain, editors, Proceedings of the Fifteenth Australasian Fluid Mechanics Conference. The University of Sydney. Paper AFMC00196. http://www . aeromech.usyd.edu.au/15afmc/ proceedings/papers/AFMC00196.pdf C36, C41 
[12] G. D. McBain and S. W. Armfield. Two-point boundary value problems, Green's functions, and product integration. ANZIAM J., 46(E):C245-C259, 2005. http://anziamj.austms.org.au/V46/CTAC2004/Mcba C36, C37, C38

[13] A. Shapiro and E. Fedorovich. Unsteady convectively driven flow along a vertical plate immersed in a stably stratified fluid. J. Fluid Mech., 498:333-352, 2004. doi:10.1017/S0022112003006803 C42

[14] J. A. C. Weideman and S. C. Reddy. A Matbab differentiation matrix suite. ACM Trans. Math. Software, 26(4):465-519, 2000.

MathReview:1939962 C41, C42 


\section{Author addresses}

1. G. D. McBain, School of Aerospace, Mechanical and Mechatronic Engineering, The University of Sydney, Australia. mailto:geordie.mcbain@aeromech.usyd.edu.au

2. S. W. Armfield, School of Aerospace, Mechanical and Mechatronic Engineering, The University of Sydney, Australia. 